\title{
A Politica Nacional de Plantas Medicinais e Fitoterápicos: construção, perspectivas e desafios
}

I 1 Climério Avelino de Figueredo, ${ }^{2}$ Idê Gomes Dantas Gurgel,

${ }^{3}$ Garibaldi Dantas Gurgel Junior I

Resumo: Em 2006, foram criadas a Política Nacional de Práticas Integrativas e Complementares e a Política Nacional de Plantas Medicinais e Fitoterápicos, para o Sistema Único de Saúde, resultado de um longo processo de demanda e construção de uma política para o setor. Este trabalho objetiva analisar a construção da política para a implantação/implementação da Fitoterapia no SUS, das facilidades e dificuldades envolvidas neste processo e dos desafios e perspectivas. Para isto foi feito a análise de documentos do Ministério da Saúde que são atinentes à questão, de artigos que abordam a política, além de artigos sobre aspectos da Fitoterapia que têm importância em relação ao seu uso nos serviços de saúde. Desta análise, observa-se que, apesar de o governo federal ter desenvolvido diversas ações, a implementação da política pouco avançou em função das dificuldades para seu uso no SUS, como o pouco conhecimento que os profissionais de saúde têm sobre a Fitoterapia, o entendimento deturpado sobre a eficácia e a segurança deste tratamento por parte de usuários e profissionais de saúde, a dificuldade do acesso à planta medicinal e ao fitoterápico, além da estruturação dos serviços nos moldes que favorecem o uso do medicamento sintético. No entanto, vê-se que esta política é importante por oferecer outra forma de tratamento, pelo fato de as plantas medicinais serem acessíveis à população, por resgatar o conhecimento popular, por favorecer a participação popular etc. e que são fatores facilitadores de sua implementação.

> Palavras-chave: políticas de saúde; implementação, Sistema Único de Saúde; fitoterapia; plantas medicinais.

\author{
1 Departamento de Fisiologia \\ e Patologia da Universidade \\ Federal da Paraíba. João Pessoa \\ Brasil. Endereço eletrônico: \\ climerioaf@bol.com.br \\ ${ }^{2}$ Centro de Pesquisa Aggeu \\ Magalhães, Fundação Oswaldo \\ Cruz. Recife, Brasil. Endereço \\ eletrônico: ideg@cpqam. \\ fiocruz.br \\ ${ }^{3}$ Centro de Pesquisa Aggeu \\ Magalhães, Fundação Oswaldo \\ Cruz. Recife, Brasil. Endereço \\ eletrônico: gurgel@cpqam. \\ fiocruz.br
}

Recebido em: 31/05/2013 Aprovado em: 11/04/2014 


\section{Introdução}

No ano de 2006, através do Decreto da Presidência da República no ${ }^{\circ}$ 5.813, de 22 de junho, foi criada a Política Nacional de Plantas Medicinais e Fitoterápicos. No mesmo ano, através de portaria do Ministério da Saúde GM/MS no 971, já havia sido criada a Política Nacional de Práticas Integrativas e Complementares no SUS (PNPIC), abrangendo, além da Fitoterapia, a Homeopatia, a Medicina Tradicional Chinesa/Acupuntura, o Termalismo/Crenoterapia e a Medicina Antroposófica. Essas duas políticas incrementaram a discussão sobre a oportunidade, a importância, as dificuldades, as facilidades e as vantagens da implementação da Fitoterapia nos serviços de saúde do SUS, e sobre as diferentes visões a respeito de como isso deve ocorrer.

A criação de uma política de âmbito nacional para o uso das plantas medicinais e dos fitoterápicos foi resultado de uma luta que remonta à época anterior à criação do SUS, em que diversos atores, como pesquisadores, gestores, profissionais de saúde e usuários tiveram papel fundamental (BRASIL, 2006a). A implementação da Fitoterapia no SUS representa, além da incorporação de mais uma terapêutica ao arsenal de possibilidades de tratamento à disposição dos profissionais de saúde, o resgate de uma prática milenar, onde se imbricam o conhecimento científico e o conhecimento popular e seus diferentes entendimentos sobre o adoecimento e as formas de tratá-lo. Pelo fato de o uso da Fitoterapia se embasar nesses dois tipos de conhecimento, aparentemente divergentes, resultam entendimentos diferentes sobro seu uso.

O Brasil tem uma rica história de uso das plantas medicinais no tratamento dos problemas de saúde da população, uso este construído com base na experiência e transmitido de forma oral (BRUNING et al., 2012). Largamente usada até meados do século XX, a Fitoterapia entrou em declínio com a intensificação do uso dos medicamentos industrializados (BRUNING et al., 2012). Com o crescente desenvolvimento da química, novas substâncias foram isoladas em laboratório e delas novos produtos de síntese surgiram, levando à paulatina substituição do uso das plantas pelo uso dos medicamentos sintetizados em laboratório, o que ocorreu de forma intensa na segunda metade do século XX (YUNES; CECHINEL FILHO, 2001), quando se consolidou a indústria farmacêutica.

Além disso, no século XX, houve, em diversos países, intenso trabalho de desqualificação do saber popular sobre as plantas medicinais (FIGUEREDO, 
2011; CARLINI, 1983), como a proibição da sua indicação por pessoas leigas e até mesmo por médicos (CHEVALLIER, 1996). Esta ofensiva contra a Fitoterapia não se fundamentou apenas na susposta inferioridade da eficácia e da segurança da planta medicinal em comparação com o medicamento sintético, nem da imprecisão e da objetividade limitada do saber popular em comparação com o saber científico (LAKATOS; MARCONI, 2001). Interesses mercantilistas, cada vez mais presentes no setor saúde, tiveram importante papel na desvalorização do uso da Fitoterapia (FIGUEREDO, 2011).

A partir da explicitação desse contexto histórico, podemos melhor situar a formulação e a implementação da política de plantas medicinais e de fitoterápicos. Esta política representa o reconhecimento do avanço na comprovação científica da eficácia e da segurança das plantas medicinais e dos medicamentos fitoterápicos, o saber popular neste campo, e também constata que o uso da terapêutica centrada no uso de medicamentos sintéticos não cumpriu a promessa implícita e explícita de dar conta do tratamento das doenças, pelos altos custos, pelos significativos efeitos adversos que têm os medicamentos sintéticos, pelos resultados nem sempre satisfatórios, o que tem levado grande número de pessoas a buscar formas alternativas de tratamento menos agressivas (BRUNING et al., 2012).

\section{Percurso metodológico}

Este trabalho constitui um estudo descritivo pautado na abordagem qualitativa que visa analisar a formulação e a implementação da Política Nacional de Plantas Medicinais e de Fitoterápicos. Ele abrange o período que se inicia no ano de 2003, quando começou o processo de discussão da política por determinação do Ministério da Saúde, e os dias atuais. Para a sua realização, foi feito inicialmente levantamento de documentos oriundos do Ministério da Saúde relacionados com a política de Fitoterapia no SUS, impressos ou virtuais, de livros e material didático sobre a Fitoterapia e de artigos disponíveis em bases eletrônicas, como: Scielo, Lilacs, Google Acadêmico e Medline.

Para a busca dos artigos nas bases eletrônicas, foram utilizados descritores como Fitoterapia, plantas medicinais, Fitoterapia no SUS, experiências de uso da Fitoterapia, Fitoterapia na atenção básica. Dos artigos encontrados, foram selecionados aqueles que abordam a implementação da política, experiências de uso da Fitoterapia nos serviços de saúde ou aspectos da Fitoterapia que 
têm importância para sua legitimação juntos aos diversos atores envolvidos na implementação da política. Foram descartados os artigos que abordam aspectos da Fitoterapia que não estão relacionados à política e às experiências de uso ou de aspectos que não têm importância direta para a politica, como aqueles que tratam dos constituintes químicos das plantas medicinais, indicações terapêuticas e levantamento de espécies vegetais utilizadas por determinadas populações.

Para a análise, deu-se ênfase aos textos que tratam da PNPIC e da PNPMF, que propõem a política para o setor, ao Relatório de gestão da PNPIC e ao informe do Ministério da Saúde sobre as ações realizadas no sentido de implementar a Fitoterapia no SUS. Os demais documentos oficiais e os artigos forneceram elementos adicionais à análise, principalmente os artigos, por trazerem elementos que apontam diversos aspectos importantes no processo de implementação.

A análise procurou fundamentar a discussão sobre a construção da política de implementação da Fitoterapia no SUS, identificando o itinerário da construção, as dificuldades para a implementação, as diretrizes propostas e os fatores presentes nesse contexto que favorecem a implementação.

\section{Resultados e discussão}

\section{A construção da política de Fitoterapia no SUS}

Do ponto de vista legal, o momento que demarca o início do processo de inserção da Fitoterapia nos serviços de saúde ocorreu em 1988, quando a Comissão Interministerial de Planejamento e Coordenação (CIPLAN), através da Resolução $\mathrm{n}^{\mathrm{o}}$ 08, disciplinou a introdução da Fitoterapia nos serviços de saúde (BRASIL, 1988b; BRASIL, 2006a). Na mesma ocasião, a CIPLAN, através de resoluçooes específicas, também disciplinou a inserção de outras práticas ditas naturais, alternativas ou integrativas como a Homeopatia e a Acupuntura (BRASIL, 1988b), fato este favorecido pela ebulição de ideias e pelo ímpeto de mudanças que estavam presentes no país, que vivia o processo de redemocratização, no campo político, e de implementação de modificações estruturais e de procedimentos no campo da saúde, sob forte influência do movimento da Reforma Sanitária, cuja culminância foi a $8^{\text {a }}$ Conferência Nacional de Saúde.

A publicação desta resolução foi um fato importante nesse processo, por ser um reconhecimento formal desta prática terapêutica como eficaz no tratamento dos problemas de saúde da população e porque ela detalhou os vários aspectos 
envolvidos na implantação. Ela considerou que a Fitoterapia poderia ser usada amplamente nos serviços de saúde, nos ambulatórios e nos hospitais, inclusive nas diversas especialidades médicas, desde que o médico tivesse a devida capacitação (BRASIL, 1988b; BRASIL, 2006a). No entanto, a implantação dessas terapêuticas foi dificultada por diversos fatores, como: a organização dos serviços no modelo tradicional, o desconhecimento dos profissionais de saúde acerca da Fitoterapia, o pouco interesse dos gestores, entre outros (FIGUEREDO, 2011).

A Conferência Nacional de Saúde, instância máxima de deliberação de políticas de saúde, seguidamente recomendou a implantação da Fitoterapia e de outras práticas integrativas e complementares no SUS. Esta recomendação está colocada explicitamente nos relatórios da $8^{\mathrm{a}}(1986), 10^{\mathrm{a}}(1996), 11^{\mathrm{a}}(2000)$ e $12^{\mathrm{a}}$ (2003) (BRASIL, 2006b).

Na mesma direção, conferências setoriais, como a $1^{\text {a }}$ Conferência Nacional de Vigilância Sanitária (2001), a 1a Conferência Nacional de Assistência Farmacêutica (2003) e a 2a Conferência Nacional de Ciência, Tecnologia e Inovação em Saúde (2004), também enfatizaram a necessidade de implantar essas práticas integrativas e complementares no SUS e incentivar seu ensino e sua pesquisa, como forma de respaldar seu uso (BRASIL, 2003; BRASIL, 2006b). Em âmbito mundial, a Organização Mundial da Saúde (OMS) tem recomendado o uso das medicinas tradicionais por seus países-membros (WHO, 2002; BRASIL, 2006b).

Anteriormente, no ano de 2004, foram aprovadas pela Agência Nacional de Vigilância Sanitária (ANVISA) quatro resoluções relacionadas com a Fitoterapia, que ajudaram na sua legitimação. São elas: Resolução de Diretoria Colegiada (RDC) no 48/2004, que dispõe sobre o registro de medicamentos fitoterápicos; Resolução-RE no 88/2004, que estabelece a lista de referências bibliográficas para avaliação de segurança e eficácia de fitoterápicos; Resolução-RE no 89/2004, que estabelece a lista de registro simplificado de fitoterápicos, e Resolução-RE n ${ }^{\circ}$ 90/2004, que estabelece um guia para a realização de estudos de toxidade préclínica de fitoterápicos (FIGUEREDO, 2011). Estas quatro resoluçôes tratam dos procedimentos para o registro de fitoterápicos e estabelecem critérios que garantem a segurança e a eficácia dos medicamentos à base de plantas medicinais e sua adequada fabricação.

A Resolução RE no 90/2004 padroniza os estudos toxicológicos préclínicos, importante passo para a realização dos demais estudos que atestarão 
a eficácia e a segurança do fitoterápico (BRASIL, 2010a). Essa regulamentação possibilitou o incremento do desenvolvimento, do registro e da comercialização de fitoterápicos, levando ao aumento de seu uso e à legitimação da Fitoterapia (FIGUEREDO et. al., 2011). Em 2005, havia 512 fitoterápicos registrados na ANVISA (CARVALHO et al., 2008).

Essas resoluções foram substituídas por outras que incorporaram modificações atinentes ao desenvolvimento do objeto por elas tratado e constituem importantes instrumentos na consolidação da Fitoterapia, por disciplinarem o registro dos medicamentos fitoterápicos, requisito para sua produção e comercialização (FIGUEREDO et al., 2011; BRASIL, 2010a).

Nos anos de 2003 e 2004, com a criação de um grupo de trabalho para discutir a implantação da Fitoterapia no SUS e a realização de um levantamento dos serviços de saúde que a utilizavam, por estados da federação e por municípios, ocorreu um rico processo de discussão com os diversos atores envolvidos neste campo na elaboração de uma política nacional para as práticas integrativas e complementares, com destaque para a Fitoterapia, que resultou na elaboração de uma proposta de política (BRASIL, 2006a, 2011b).

Aprovada no âmbito do Ministério da saúde, a proposta de uma política nacional para as práticas integrativas, nas quais se inclui a Fitoterapia, foi encaminhada ao Conselho Nacional de Saúde (CNS), em 2004. No entanto, sua aprovação no CSN foi muito demorada, em razão de divergências entre os representantes das diversas categorias de profissionais de saúde a respeito da questão de quem poderia praticar estas terapêuticas no SUS.

Após a aprovação no CNS, em fevereiro de 2006, com a denominação de Política Nacional de Práticas Integrativas e Complementares (PNPIC) no SUS (BRASIL, 2006a), a luta política se transferiu do CNS para o âmbito do Ministério da Saúde (MS), daí porque somente em maio e junho de 2006, a proposta aprovada transformou-se na Política Nacional de Práticas Integrativas e Complementares no SUS (PNPIC) e na Política Nacional de Plantas Medicinais e Fitoterápicos (PNPMF), esta mais abrangente (BRASIL, 2011b), do que aquela no que diz respeito à Fitoterapia, por definir objetivos, diretrizes mais amplas, formas de monitoramento e avaliação e responsabilidades institucionais (BRASIL, 2006a). 
Essas políticas têm como objetivo garantir a prevenção de agravos, a promoção e a recuperação da saúde com ênfase na atenção básica à saúde e visa contribuir para o aumento da resolubilidade do sistema com qualidade, eficácia, eficiência, segurança, sustentabilidade, controle e participação social (BARROS, 2006; BRASIL, 2011b).

\section{A importância da Fitoterapia para o SUS}

A existência de uma política nacional para a Fitoterapia no SUS tem grande importância para o país, considerando o que propõe esta política e o contexto econômico, social, cultural, científico e sanitário presentes no Brasil. Uma das diretrizes da PNPMF e da PNPIC é o Incentivo à pesquisa e ao desenvolvimento de plantas medicinais e de fitoterápicos, priorizando a biodiversidade do país (BRASIL, 2006a; BRASIL, 2006b).

O Brasil é um país de grande diversidade de espécies vegetais, com muitas plantas medicinais que são matérias-primas para a fabricação de fitoterápicos e outros medicamentos. As espécies vegetais com poderes medicinais são numerosas (FIRMINO; BINSFELD, 2013). Isto é comprovado por séculos de uso pela população, não apenas a partir do início da colonização, mas antes dela, pelos habitantes nativos que tinham nas plantas medicinais o principal meio de cura de suas enfermidades (BRUNING et al., 2012).

Com o desenvolvimento da Química, foram isolados muitos princípios ativos de plantas que resultaram em medicamentos (CHECHINEL FILHO; YUNES, 1998). Atualmente, com a consolidação dos estudos pré-clínicos e clínicos sobre as plantas medicinais, sua eficácia e sua segurança, que antes eram comprovadas, de forma limitada, pelo uso popular, passaram também a sê-lo pelos estudos científicos.

A criação de uma política para a Fitoterapia abre perspectivas de desenvolvimento de estudos com as plantas medicinais, o que pode dará ao Brasil papel de destaque no cenário mundial na produção de medicamentos fitoterápicos, um mercado que cresce percentualmente mais do que o mercado de medicamentos tradicionais (CARVALHO, 2006). Neste sentido, em 2009, foi realizado o Seminário "Complexo Industrial da Saúde e Fitoterápicos", com a finalidade de integrar diversos setores afins à questão como o setor industrial, os 
serviços de saúde, a academia e o Ministério da Saúde e identificar e desenvolver as potencialidades para produção de fitoterápicos (BRASIL, 2012).

Além da possibilidade que se abre na área de produção de fitoterápicos, a criação da PNPMF se justifica por outras razões, como: possibilita aos profissionais de saúde outra forma de tratamento; os custos financeiros do uso de plantas medicinais e fitoterápicos são menores; as plantas medicinais e os fitoterápicos têm menor potencial de provocar efeitos adversos; facilidade de acesso às plantas medicinais; inserção cultural da Fitoterapia nos usos e costumes da população; garantia do direito do usuário de escolher outra forma de tratamento, se assim preferir; incremento da participação popular no SUS, resgate do conhecimento popular; criação de emprego e renda nos municípios que implementarem a Fitoterapia, devido à cadeia produtiva que se estabelece para a produção das plantas medicinais e dos fitoterápicos etc. (FIGUEREDO, 2011).

\section{A implementação da Fitoterapia no SUS: diretrizes, facilidades e dificuldades}

Tendo em vista a importância da implementação da Fitoterapia no SUS e a existência de muitas dificuldades para que isto ocorra, a PNPMF e a PNPIC elencam uma série de diretrizes para a superação das dificuldades e potencialização de fatores que facilitam esse processo (BRASIL, 2006a; BRASIL, 2006b). A seguir, discutiremos algumas dificuldades e fatores facilitadores, relacionando-os com as diretrizes das duas políticas.

Um dos aspectos importantes a ser ressaltado nesse processo é o papel do usuário (BRASIL, 2011b). Apesar da disseminação do uso do medicamento sintético, ainda é muito alto o percentual daqueles que usam as plantas medicinais, isoladamente ou em conjunto com ele, e isto é feito com base no conhecimento popular (BRUNING et al., 2012). Isto ocorre por diversas razões, como a crença de que elas têm menos efeitos adversos.

Muitas situações de adoecimento são tratadas sem se buscar os serviços de saúde, e quando isto ocorre nem sempre há o fornecimento de medicamentos após a prescrição e as pessoas não dispõem de recursos para comprá-los. No entanto, o uso popular das plantas medicinais, por falta de informações dos usuários, nem sempre é feito de forma correta no que diz respeito à indicação, à qualidade da matéria-prima vegetal utilizada e ao preparo das formulações caseiras. E erros 
em um ou em alguns destes aspectos podem não só comprometer a eficácia do tratamento, mas acarretar danos.

Muitas plantas medicinais têm efeitos adversos e podem provocar interações com outras plantas ou com medicamentos sintéticos (SILVEIRA et al., 2008). Disto resulta a necessidade da disseminação de informações a respeito do uso correto das plantas medicinais, com especificidades para cada público a que se destina: gestores, profissionais de saúde, usuários etc. (BRASIL, 2006b). A falta de informação leva à distorção das possibilidades terapêuticas da Fitoterapia.

Pelo fato de o uso de plantas medicinais ser arraigado na tradição popular e a população ser depositária de amplo conhecimento sobre elas, uma questão importante que a PNPMF estabelece é o reconhecimento da valorização do conhecimento popular. A diretriz no 10 da PNPMF recomenda: "Promover e reconhecer as práticas populares de uso de plantas medicinais e 'remédios caseiros" (BRASIL, 2006a, p. 22).

No meio popular, é comum o uso de uma mesma planta para um grande número de doenças e sintomas e a crença de que elas podem curar até mesmo doenças graves, como o câncer. Segundo os pesquisadores da ação terapêutica das plantas medicinais, embora haja esta possibilidade, o uso das plantas medicinais deve ser criterioso e muitas vezes seu papel é complementar ao uso do medicamento sintético.

Esta inadequação do uso popular fortalece o posicionamento de muitos profissionais de saúde de que o uso da Fitoterapia não traz muitos benefícios; daí porque, para esses, as plantas medicinais deveriam tão somente ser usadas com a finalidade de fornecer princípios ativos para a produção de fitofármacos. Este posicionamento advém da desinformação da potencialidade de cura das plantas medicinais e da negação da importância do conhecimento popular no uso da Fitoterapia, constituindo uma dificuldade para a implementação.

A formação dos profissionais de saúde, principalmente do médico, ocorre dentro de uma concepção de que o conhecimento científico é o único confiável por ele ser objetivo, sistemático, crítico, reprodutível (LAKATOS; MARCONI, 2001).

O conhecimento popular sobre o processo saúde-doença e suas diversas formas de manifestação não é valorizado; muitas vezes, sequer é escutado. Se isto é mais facilmente imposto quando a fala do paciente é sobre aspectos clínicos do seu adoecimento, em relação ao uso de plantas medicinais isto é mais difícil, tendo 
em vista que, durante séculos, o conhecimento sobre elas foi majoritariamente construído com base no uso popular.

Em relação a esse conhecimento, profissionais de saúde geralmente têm uma atitude desfavorável consoante as representações mais amplas da classe médica (ROSA et al., 2011). Em função disto, há a necessidade do estabelecimento de uma interação entre o conhecimento popular e o conhecimento científico. Segundo a PNPIC, é necessário:

Resgatar e valorizar o conhecimento tradicional e promover a troca de informações entre grupos de usuários, detentores deconhecimento tradicional, pesquisadores, técnicos, trabalhadores em saúde e representantes da cadeia produtiva de plantas medicinais e fitoterápicos. (BRASIL, 2006b, p. 50).

Quando as plantas medicinais passam a fazer parte do arsenal terapêutico à disposição do profissional de saúde, principalmente o médico, é necessário que este interaja com o usuário a respeito do que ele sabe a respeito das plantas medicinais e como ele as usa.

Sobre muitas plantas já há grande acúmulo de estudos científicos que atestam sua eficácia e segurança. E geralmente há uma concordância entre o que apontam estes estudos e o que indica o uso popular. Por isso, quando se busca estudar plantas para determinada indicação terapêutica, o principal critério de escolha é seu uso popular (CHECHINEL FILHO; YUNES, 1998). No entanto, dada a grande quantidade de plantas que são usadas pela população, sobre muitas delas não há estudos científicos ou eles são incipientes, o que gera receio dos profissionais de saúde em prescrevê-las, com base no conhecimento popular, visto que, segundo Rosa et al. (2011), a medicina baseada em evidência é a norteadora da prática médica.

A Resolução de Diretoria Colegiada (RDC) 14/2010 da Agência Nacional de Vigilância Sanitária (ANVISA), que trata do registro de medicamentos fitoterápicos, estabelece três formas pelas quais devem ser comprovadas a eficácia e a segurança dos medicamentos fitoterápicos: estudos pré-clínicos e clínicos, sustentação na literatura enumerada na Instrução Normativa 05/2010 da ANVISA e tradicionalidade de uso, isto é o uso popular. Esta é analisada com base em seis critérios, sendo um deles o uso continuado pela população por um período não inferior a 20 anos (FIGUEREDO et al., 2011; BRASIL, 2010a).

A possibilidade de registro dos medicamentos fitoterápicos com base no conhecimento popular tem sido objeto de críticas de setores da academia e dos 
profissionais de saúde que acham que este conhecimento não é suficiente para

aferir segurança e eficácia das plantas, reeditando uma disputa antiga entre médicos e curandeiros de variados tipos. Para outros, o conhecimento popular deve ser valorizado, atualizado e disseminado, e com o conhecimento científico deve haver uma complementaridade e não uma disputa (BRASIL, 2006b).

Ainda em relação ao usuário, a PNPMF abre espaço para a participação popular, um dos princípios norteadores do SUS (BRASIL, 2003; BRASIL, 1990). Sendo a população detentora de extenso conhecimento sobre as plantas medicinais, numa perspectiva de troca de conhecimento e experiência sobre seu uso, pode haver aproximação entre a população e os serviços de saúde que dispõem desse tipo de tratamento. Há, pois, reforço a este princípio que não tem sido concretizado a contento, embora a lei orgânica da saúde preveja mecanismos para seu exercício, como é o caso dos conselhos de saúde, nas três esferas de poder (BRASIL, 1990).

Nos serviços onde a Fitoterapia já foi implementada, é comum a participação dos usuários em atividades como palestras, oficinas, construção de canteiros etc. Isto ocorre porque eles se sentem sujeitos ativos, por deterem conhecimento na área e por serem chamados a participar de atividades em que se sentem capazes de interagir, coisa que não ocorre no dia a dia dos serviços, quando eles operam apenas com os tratamentos tradicionais.

Outra vantagem da implementação da Fitoterapia diz respeito à facilidade do seu acesso. $\mathrm{O}$ tratamento fitoterápico pode ser feito através do medicamento industrializado, do medicamento manipulado (BRASIL, 2010b) ou através do uso da planta, mediante preparaçôes caseiras.

$\mathrm{O}$ medicamento fitoterápico industrializado tem preço parecido com os medicamentos sintéticos, o que dificulta o acesso a ele. Desde 2008, está incluído na relação de medicamentos da assistência farmacêutica na atenção básica. Inicialmente, foram incluídos fitoterápicos de apenas duas plantas (BRASIL, 2011b). Atualmente, fitoterápicos de 11 plantas estão relacionados, podendo ser adquiridos com recursos destinados à assistência farmacêutica na atenção básica, mediante pactuação (BRASIL, 2012), em consonância com diretrizes da PNPIC e PNPMF, que preconizam a facilitação do acesso à planta medicinal e ao fitoterápico.

Apesar de ser uma medida positiva, ela é muito tímida, tendo em vista que a relação de medicamentos sintéticos é bem extensa e que todos os medicamentos 
homeopáticos inscritos na farmacopeia homeopática podem ser comprados com esses recursos (BRASIL, 2011b). Isso mostra uma incoerência entre o que está previsto na assistência farmacêutica na atenção básica e as recomendações da PNPIC, de:

Ampliação da oferta de fitoterápicos, por intermédio de farmácias públicas com manipulação de fitoterápicos, que atenda a demanda e as necessidades locais, respeitando a legislação pertinente às necessidades do SUS na área. (BRASIL, 2006b, p. 47).

Alternativa para o uso do medicamento fitoterápico é fazer sua manipulação em "oficinas" estruturadas nos municípios, visto que elas não exigem equipamentos e processos sofisticados. Esta possibilidade foi regulamentada com a criação das Farmácias Vivas, através da Portaria MS no 886, de 22 de abril de 2010 (BRASIL, 2010b).

Para a manipulação de fitoterápicos em "oficinas", a matéria-prima vegetal utilizada pode ser adquirida junto a fornecedores ou pode ser produzida pelo próprio município. Neste sentido, em 19 de junho de 2012, foi publicada a Portaria $\mathrm{n}^{\circ} 13$, que habilitou 12 municípios, selecionados pelo Edital SCTIE no 1 , de 26 de abril de 2012, para receberem recursos para estruturação, consolidação e fortalecimento de Arranjos Produtivos Locais (APLs), no âmbito do SUS, conforme estabelecem a PNPMF e o Programa Nacional de Plantas Medicinais e de Fitoterápicos (BRASIL, 2012). Esses APLs são fontes de emprego e renda, algo positivo para diminuir a pobreza, notadamente em regiōes com menor grau de desenvolvimento.

Devido a esses fatores, o medicamento fitoterápico manipulado tem baixo custo, algo de muita importância por facilitar o acesso dos usuários ao medicamento, diferentemente dos medicamentos sintéticos, cujos insumos têm alto custo, o que se reflete no seu preço final.

Além do uso do medicamento manipulado, há outra possibilidade que é a mais utilizada pela população: as preparações caseiras à base das plantas medicinais. Desde que usadas com a orientação do profissional de saúde, as preparações caseiras são capazes de resolver muitas situações de adoecimento que são corriqueiras. A isso se acrescenta a facilidade de acesso às plantas, a qualquer hora e em qualquer lugar. Normalmente, as pessoas têm, no entorno de suas casas, espécies vegetais plantadas. Por isto, tão logo apareça o problema de saúde, ele pode ser tratado 
através de formulações caseiras (SANTOS et al., 2011). E a precocidade do tratamento é um dos importantes fatores para o êxito do tratamento.

Um dos pontos de estrangulamento do SUS é o provimento do medicamento ao usuário, devido a seu elevado custo. $\mathrm{O}$ incremento do uso das plantas medicinais poderá amenizar este problema (BRASIL, 2011b). Mas em desacordo ao que estabelece a política e o programa de plantas medicinais e de fitoterápicos (BRASIL, 2007; BRASIL, 2006a) poucas ações foram feitas para que fosse incentivado o plantio de espécies vegetais no entorno domiciliar, bem como a capacitação de pessoas da comunidade para lidar com o seu manejo.

Outro problema a considerar é o pequeno número de profissionais de saúde que têm conhecimentos suficientes para prescrever plantas medicinais e medicamento fitoterápicos. Embora a população use constantemente a Fitoterapia com base nos conhecimentos populares, os profissionais de saúde, para dela se utilizarem, necessitam ter conhecimento mais aprofundado e até mesmo o conhecimento popular lhe é deficiente, pois são oriundos de extratos sociais que usam quase exclusivamente o medicamento sintético.

Mesmo com o significativo incremento do uso e do estudo da Fitoterapia, ainda é incipiente o ensino desta forma de tratamento nos cursos de graduação. Como resultado, os profissionais dos serviços de saúde, majoritariamente, têm deficiência neste campo do conhecimento (ROSA et al., 2011; SANTOS, 2011). E o desconhecimento é terreno fértil para crescer o preconceito a respeito da Fitoterapia, comumente vista como tratamento eficaz apenas para situações de adoecimento superficial.

Para aumentar o conhecimento dos profissionais de saúde, a PNPIC recomenda: "Estimular as universidades a inserir nos cursos de graduação e pós-graduação, envolvidos na área, disciplinas com conteúdo voltado às plantas medicinais e fitoterapia” (BRASIL, 2006b, p. 49).Idêntico entendimento é encontrado na PNPMF, já que uma de suas diretrizes é: "Incentivar a formação e a capacitação de recursos humanos para o desenvolvimento de pesquisas, tecnologias e inovação em plantas medicinais e fitoterápicos" (BRASIL, 2006a,p. 21).

Enquanto o ensino da Fitoterapia na graduação e na pós-graduação não produz os resultados esperados, ou seja, profissionais de saúde com conhecimento da Fitoterapia suficiente para usá-la nos serviços, medidas pontuais são tomadas, 
como a elaboração do Formulário Fitoterápico. Além do valor simbólico de o formulário ser inserido na Farmacopeia Brasileira, ele fornece um conjunto de formulações de plantas medicinais que têm estudos científicos comprobatórios de sua eficácia e sua segurança, com largo uso pela população, além de elas contemplarem as principais situaçóes de adoecimento (BRASIL, 2011a). Sendo o formulário chancelado por um grupo de especialistas na área, sua utilização por parte dos profissionais pode ser feita sem que eles tenham maior aprofundamento, o que pode levar ao incremento do uso.

Em consonância com a PNPIC e com a PNPMF, o Ministério da Saúde financiou a capacitação de 440 farmacêuticos em Gestão em Fitoterapia e Homeopatia, nos cursos de Pós-Graduação (lato sensu) em Gestão da Assistência Farmacêutica, voltados para o Sistema Único de Saúde, em 13 instituições de ensino superior/escolas de Saúde Pública (IES/ESP) do país. Outra medida foi a realização de um curso à distância sobre Fitoterapia para médicos do SUS, que teve início em janeiro de 2012 e contou com a participação de 300 médicos de todo o Brasil (BRASIL, 2012).

Em relação aos gestores, o desconhecimento a respeito da Fitoterapia faz com que eles não busquem implementar esta forma de tratamento. Muitos sequer têm conhecimento da existência da política para o setor. Outros criam obstáculos quando há profissionais de saúde que, por conta própria, utilizam a Fitoterapia como tratamento complementar. Para a gestão, muitos levam a mesma postura de desconhecimento, desconfiança e descrédito que têm como profissionais de saúde em relação à Fitoterapia.

Além disso, a implementação da Fitoterapia implica o rearranjo do modo de operação dos serviços de saúde, a capacitação de profissionais de saúde nesta área, fornecimento da planta medicinal ou do medicamento fitoterápico ao usuário, para o que se necessita de recursos financeiros e tempo, algo escasso no SUS, embora no médio e no longo prazos a implementação traga vários benefícios.

Para enfrentar este problema, o Ministério da Saúde, por meio do Departamento de Assistência Farmacêutica e Insumos Estratégicos da Secretaria de Ciência, Tecnologia e Insumos Estratégicos, em parceria com a Fundação Oswaldo Cruz (Fiocruz), têm apoiado municípios e estados na estruturação da assistência farmacêutica com plantas medicinais e com fitoterápicos. Em uma primeira fase, 24 municípios e quatro estados foram contemplados, com uma 
oficina para gestores, tendo como objetivo a estruturação de projeto. $\mathrm{Na}$ segunda fase, 12 municípios e dois estados deram continuidade ao projeto com a aquisição de bens de consumo e de capital (BRASIL, 2012).

O desconhecimento ou o conhecimento deturpado a respeito do uso de plantas medicinais e medicamentos fitoterápicos ocorre não apenas entre profissionais de saúde e os gestores, mas também em parcelas dos usuários. Apesar do largo uso que as pessoas fazem das plantas medicinais e da extensão do conhecimento popular, de forma individualizada, o conhecimento das pessoas sobre as plantas medicinais é cheio de imprecisões, principalmente no que diz respeito à forma como são feitas as preparações caseiras, as indicaçôes das plantas e o alcance do uso da Fitoterapia. Tais imprecisões podem acarretar o fracasso do tratamento, criando um comportamento de recusa a usar as plantas medicinais em determinadas situações de adoecimento.

Há uma percepção arraigada nas pessoas de que as plantas medicinais são muito úteis no tratamento de doenças corriqueiras, de menor gravidade e de curso rápido, como gripes, problemas digestivos leves, infecções da boca e orofaringe, infecções de pele etc. Neste caso, elas usam as plantas com base em conhecimento próprio ou por sugestão de familiares, vizinhos e amigos. Como consequência desta percepção, quando o médico do serviço de saúde prescreve plantas medicinais para situações de adoecimento consideradas mais severas, o paciente não fica satisfeito, pois para ele, para ser tratado com a Fitoterapia não justificaria ter procurado o serviço. Ele mesmo teria se tratado em casa, já que dispóe do conhecimento sobre o uso das plantas medicinais. Este comportamento de recusa do uso da Fitoterapia no tratamento de doenças tidas como mais complexas é um desafio que os profissionais de saúde devem superar para que esse tratamento possa ser utilizado de forma mais abrangente.

Daí porque tanto a PNPIC quanto a PNPMF têm nas suas diretrizes a socialização de informações sobre as plantas medicinais e medicamento fitoterápicos, com a devida adequação aos diferentes segmentos do público-alvo, com destaque para os usuários (BRASIL, 2006a; BRASIL, 2006b). No entanto, é o profissional de saúde o ator privilegiado na disseminação da informação para os usuários, notadamente durante os momentos de atendimento, mas, como referido anteriormente, o profissional de saúde ainda tem muita resistência ao uso da Fitoterapia (SANTOS, 2011) e não tem a prática de repassar informações durante a consulta. 
Uma questão crucial para a implementação da Fitoterapia é seu financiamento. Todo o Sistema Único de Saúde está estruturado no modelo que usa o medicamento sintético. A implementação da Fitoterapia implica novos custos. Embora a Fitoterapia seja uma forma de tratamento de baixo custo, a estruturação desta forma de tratamento pressupõe investimentos iniciais de monta, como capacitação dos profissionais da rede, criação de laboratórios de manipulação de medicamentos fitoterápicos, estruturação de uma cadeia produtiva capaz de fornecer matéria-prima vegetal, ou seus derivados, com qualidade (FIGUEREDO, 2011). Para isso, segundo a PNPIC, é necessário que haja o: "estabelecimento de política de financiamento para o desenvolvimento de ações voltadas à implantação das plantas medicinais e da fitoterapia no SUS" (BRASIL, 2006b, p. 51).

Do contrário, as ações e as atividades que a política define terão que competir com outras atividades e ações dentro do SUS. Embora haja iniciativas neste sentido por parte do Ministério da Saúde, considerando a amplitude das necessidades para a implementação da política, as iniciativas ainda são pontuais, demonstrando incoerência entre os objetivos propostos e os meios para viabilizá-los.

A implementação da Fitoterapia, como qualquer política pública, precisa ser submetida a avaliações no sentido de detectar falhas e ressaltar êxitos. A PNPIC e a PNPMF propõem que ela seja submetida a avaliações para que sejam feitas as retificações necessárias (BRASIL, 2006a; BRASIL, 2006b). Os diversos segmentos existentes no setor saúde podem ter percepções diferentes a este respeito e, mesmo entre os segmentos, há diferenciações a respeito da implementação.

A avaliação da política possibilita a explicitação dos diferentes pontos de vista, e do confronto saudável das diferenças podem emergir novos rumos que venham a dar vitalidade à política.

\section{Considerações finais}

Desde a criação da Política Nacional de Plantas Medicinais e Fitoterápicos, em 2006, ocorreram muitas ações no sentido de sua implementação. Diversos estados e municípios criaram políticas locais para o setor, com a aprovação de leis específicas. Muitos serviços de saúde passaram a oferecer esse tipo de tratamento e aumentou o número de profissionais que utilizam a fitoterapia no tratamento de seus pacientes. 
Além disso, a Fitoterapia foi inserida em muitos cursos de graduação da área da saúde, e programas de pós-graduação passaram a ter as plantas medicinais como linha de pesquisa. A inserção crescente da Fitoterapia na academia favorece sua disseminação, pois aprofunda o conhecimento sobre ela, atesta a eficácia e a segurança de seu uso e lhe confere respaldo científico, diminuindo o descrédito e o preconceito que ainda é marcante entre profissionais de saúde, gestores e usuários.

Todavia, todo esse crescimento ainda não foi suficiente para torná-la uma prática frequente nos serviços de saúde. Várias dificuldades impedem que todo o potencial desta forma de tratamento seja explorado, o que seria benéfico para os usuários, para o SUS e para o Brasil. ${ }^{1}$

\section{Referências}

BARROS, N.F. Política Nacional de Práticas Integrativas e Complementares no SUS: uma ação de inclusão. Ciência e Saúde Coletiva, v. 1, n. 3, p. 850, 2006.

BRASIL. Agência Nacional de Vigilância Sanitária. Formulário de Fitoterápicos da Farmacopeia Brasileira. Brasília: Anvisa, 2011. Disponível em <www.anvisa.gov.br>. Acesso em: 30 out. 2012.

BRASIL. Ministério da Saúde. Secretaria de Atenção à Saúde. Departamento de Atenção Básica. Coordenação Nacional de Práticas Integrativas e Complementares. Relatório de Gestão: 2006/2010. Práticas Integrativas e Complementares no SUS. Brasília: Ministério da Saúde, 2011.

BRASIL. Ministério da Saúde. Fitoterapia no SUS. Disponível em: < http://portal.saude. gov.br >. Acesso em: 17 nov. 2012.

. Ministério da Saúde. Secretaria de Assistência à Saúde. Departamento de Atenção Básica. Politica Nacional de Práticas Integrativas e Complementares no SUS: PNPIC-SUS. Brasília: Ministério da Saúde, 2006.

. Ministério da Saúde. Secretaria de Ciência, Tecnologia e Insumos Estratégicos, Departamento de Assistência Farmacêutica. Programa Nacional de Plantas Medicinais e Fitoterápicos. Brasília: Ministério da Saúde, 2007. Disponível em:< http://portal.saúde.gov. br > Acesso em: 13 abr. 2009.

. Ministério da Saúde. Secretaria de Ciência, Tecnologia e Insumos Estratégicos, Departamento de Assistência Farmacêutica. Politica Nacional de PlantasMedicinais e Fitoterápicos. Brasília: Ministério da Saúde, 2006.

. Resolução CIPLAN No 8/88, de 8 de mar.1988. Diário Oficial da República Federativa do Brasil. Brasília, v. 126, n. 48, p. 3.999-4.000, 11mar. Seção I, 1988.

. Constituição da Republica Federativa do Brasil. Brasília: Senado Federal, 1988. 
Lei n. 8.080, de 19 de setembro de 1990. Dispóe sobre as condições para a promoção, proteção e recuperação da saúde, organização e o funcionamento dos serviços correspondentes e dá outras providências. Diário Oficial da República Federativa do Brasil, Brasília, DF, 20 set. 1990. Seção 1.

Ministério da Saúde. O papel da medicina natural e práticas complementares de saúde na consolidação dos princípios e diretrizes da Reforma Sanitária. Ministério da Saúde: Brasília, 2003.

Ministério da Saúde. Agência Nacional de Vigilância Sanitária. Consolidado de Normas da COFID. Brasília: Ministério da Saúde, 2010. Disponível em <htpp://www. anvisa.gov.br>. Acesso em: 30 mar. 2011.

. Ministério da Saúde. Portaria no 886, de 20 de abril de 2010. Institui a Farmácia Viva no âmbito do Sistema Único de Saúde. Brasília: Ministério da Saúde, 2010. Disponível em <http://portal.saude.gov.br>. Acesso em: 05 jan. 2013.

BRUNING, M.C.R.; MOSEGUI, G.B.G.; VIANA, C.M.M. A utilização da fitoterapia e de plantas medicinais em unidades básicas de saúde nos municípios de Cascavel e Foz do Iguaçu-Paraná: a visão dos profissionais de saúde. Ciência e Saúde coletiva, v. 17, n. 10, p. 2.675-2.685, 2012. Disponível em: http://www.scielosp.org/pdf/csc/v17n10/17.pdf. Acesso em: 10 jan. 2013.

CARLINI, E.A. Pesquisas com plantas medicinais usadas em medicina popular. Rev. Ass. Med. Bras., v. 29, p. 109-110, 1983.

CARVALHO, A.C.B. et al. Situação de registro de medicamentos fitoterápicos no Brasil. Revista Brasileira de Farmacognosia, v. 18, n. 2, p. 314-319, abr-jun. 2008.

CHECHINEL FILHO, V.; YUNES, R.A. Estratégias para a obtenção de compostos farmacologicamente ativos a partir de plantas medicinais. Conceitos sobre modificação estrutural para otimização da atividade. Química Nova, v. 21, n. 1, 1998. Disponível em: <http://www.scielo.br>. Acesso em: 04 jan. 2013.

CHEVAlliER, A. The Encyclopedia of Medicinal Plants. London: Dorling Kindersley, 1996.

FIGUEREDO, C.A. Fitoterapia (texto didático). João Pessoa: Núcleo de Estudo e Pesquisas Homeopáticas e Fitoterápicas, 2011.

FIGUEREDO, C.A.; GURGEL, I.D.G.; GURGEL JUNIOR, G.D. A implantaçāo da Fitoterapia no SUS: uma avaliação à luz do arcabouço normativo. In: OLIVEIRA, M.H.B. et al. (Orgs.). Direito e saúde : cidadania e ética na construção de sujeitos sanitários. Maceió: EdUFAL, 2011.

FIRMINO, F.C. BINSFELD, P.C. A biodiversidade brasileira como fonte de medicamentos para o SUS. Disponível em:<http://www.cpgls.ucg.br> Acesso em: 05 jan.2013.

LAKATOS, E. M.; MARCONI, M.A. Metodologia científica. São Paulo: Atlas, 2001.

ROSA, C.; CÂMARA, S.G.; BÉRIA, J.U. Representações e intenção de uso da fitoterapia 
na atenção básica à saúde. Ciência e Saúde Coletiva. Rio de Janeiro, v. 16, n. 1, jan. 2011.

Disponível em: <http://www.scielo.br/scielo.php>. Acesso em: 05 nov. 12.

SANTOS, R.L. et al. Análise sobre a Fitoterapia como prática integrativa no Sistema Único de Saúde. Revista Brasileira de Plantas Medicinais. Botucatu, v. 13, n. 4, 2011.

SILVEIRA, Y.M.S.C.; RAMIRES, J.C.L. O uso de plantas medicinais na área urbana de Montes Claro-MG: reflexões a partir da população atendida na Estratégia Saúde da Família do bairro Morrinhos. In: Encontro Nacional de Geógrafos, XVI. Anais... Porto Alegre, 2010. Disponível em: <http://www.agb.org.br>. Acesso em: 30 dez. 2012.

SILVEIRA, P.F.; BANDEIRA, M.A.M.; ARRAIS, P.S.D. Farmacovigilância e reações adversas às plantas medicinais e fitoterápicos: uma realidade. Rev. bras. farmacogn. João Pessoa, v. 18, n. 4, oct-dez. 2008. Disponível em: < http://www.scielo.br>. Acesso em: 05 nov. 2012.

WORLD HEALTH ORGANIZATION. Traditional Medicine Strategy 2002-2005. Geneva: WHO, 2002.

YUNES, R.A.; CECHINEL FILHO, V. In: YUNES, R.A.; CALIXTO, J.B. (Org.). Plantas Medicinais sob a Ótica da Química Medicinal Moderna. Chapecó: Argos, 2001.

\section{Nota}

${ }^{1}$ C.A. de Figueiredo, I.G.D. Gurgel e G.D. Gurgel Junior participaram igualmente da concepção do projeto; análise e interpretação dos dados; e redação e revisão crítica do artigo. 


\section{The National Policy on Medicinal Plants and Phytotherapy: building, perspectives and challenges} In 2006, the National Policy on Integrative and Complementary Practices and the National Policy on Medicinal Plants and Herbal Medicines were created for the Brazilian National Health System (the SUS), as result of a long process of demand and construction of a policy for the sector. This paper aims to analyze the construction of policy for deployment / implementation of phytotherapy in SUS, the facilities and difficulties involved in this process and the challenges and prospects. So we analyzed documents from the Ministry of Health relating to the issue of articles covering politics, as well as articles on aspects of herbal medicine that have significance in relation to its use in health services. From this analysis, it is observed that, although the federal government has developed several initiatives, the implementation of the policy made little progress because of the difficulties for its use in the SUS, as the little knowledge that health professionals have on Phytotherapy, understanding misrepresented on the efficacy and safety of this treatment on the part of users and health professionals, the difficulty of access to medicinal plant and phytotherapics, besides structuring services in ways that favor the use of synthetic medication. However, we see that this policy is important for offering another form of treatment, the fact that medicinal plants are accessible to the population, for rescuing the popular understanding by encouraging popular participation etc. and are facilitating factors for their implementation.

> Key words: health policy; implementation, Unified Health System; herbal medicine; medicinal plants. 